- Original Article

\title{
Compliance and Effectiveness of Smoking Cessation Program Started on Hospitalized Patients
}

\author{
Gun Hee Shin, Sung Won Yi, Yoo Seock Cheong*, Eal Whan Park, Eun Young Choi \\ Department of Family Medicine, Dankook University College of Medicine, Cheonan, Korea
}

Background: Varenicline is now very useful medication for cessation; however, there is only little result of researches with varenicline for cessation of hospitalized patients. This research attempted to analyze the cessation effect of medication and compliance of hospitalized patients.

Methods: This research included data for 52 patients who were prescribed varenicline among 280 patients who were consulted for cessation during their admission period. This research checked whether smoking was stopped or not after six months and analyzed their compliance, the factors for succeeding in smoking cessation.

Results: One hundred and ninety hospitalized patients participated in smoking cessation counseling among 280 patients who included consultation from their admission departments. And varenicline was prescribed for only 80 patients after counseling. Nineteen smokers were successful in smoking cessation among 52 final participants representing the rating of success of $36.5 \%$. The linkage between compliance of varenicline and rate of smoking successful has no statistical significance. The factors for succeeding in smoking of hospitalized patients are admission departments, diseases, and economic states.

Conclusion: Smoking cessation program has low inpatient compliance. Cooperation of each departments is very important for better compliance. Success rate of cessation was relatively high (36.5\%). Cessation attempt during hospitalization is very effective strategy.

Keywords: Smoking; Hospitalization; Varenicline; Smoking Cessation 


\section{INTRODUCTION}

Smoking has been widely known as one of the main causes of respiratory and circulatory diseases and also various cancers. ${ }^{1)}$ However, it is not easy for smokers to quit smoking completely. There is a growing awareness in the direct and indirect detoxification of smoking. Also, most of the public health centers operate smoking cessation clinics. That is, a smoke-free environment has been formed in recent days. Nonetheless, according to the National Health and Nutrition Examination Survey for 2013, ${ }^{2)}$ the smoking rate of South Korean men aged 19 years old or older is still high at $42.5 \%$.

Being hospitalized due to a disease or accident would not be a pleasant experience for patients. However, this would be deemed as a golden opportunity for smokers to try for cessation because they find it inconvenient to smoke during this period. On that account, introducing a systematic smoking cessation program for hospitalized patients can allow smokers to turn crisis into opportunity. In the meantime, several studies have focused on the outpatient clinic smoking cessation programs, and multiple number of the overseas studies have examined the systematic smoking cessation consultation and programs for hospitalized patients. ${ }^{3,4)}$ However, there have been only a few domestic studies that examined the effects of smoking cessation programs for hospitalized patients.

Compliance is the concept to determine whether a patient's behavior is consistent enough with the prescription or advice of a medical doctor. ${ }^{5)}$ This study aimed to examine not only the drug compliance but also the performance consistency of treatment process in which a smoking cessation request would lead to an actual advice and prescription. In this regard, this study aimed to define the performance consistency of treatment process with the term treatment compliance. Unlike those smokers who visit an outpatient clinic for the purpose of quitting smoking, hospitalized patients do not usually aim to quit smoking as a top priority. On that account, it is expected that there will be a lot of obstacles for providing actual consultation and prescription on smoking cessation. The term treatment compliance, which is used in this study, is a more comprehensive concept than the drug compliance. That is, the term treatment compliance includes the ratio of how many smoking cessation requests of hospitalized patients would lead to an actual smoking cessation consultation. Also, it includes the aspect of whether patients comply properly with a visiting day to an outpatient clinic after being discharged from hospital and the aspect of whether they follow properly the behavioral changes ordered by a medical doctor or the treatment related orders.

Varenicline functions as an agonist in a nicotine receptor. In this regard, many studies have proved the effect of smoking cessation ever since varenicline had been released in South Korea. ${ }^{6-8)}$ This study was conducted for the purpose of examining the treatment compliance of those smokers referred in 'the smoking cessation program for hospitalized patients' operated by the family medicine department among the hospitalized patients in various departments of the university hospital. In addition, this study aimed to identify the effects of varenicline, which was the main medicine for them, on smoking cessation.

\section{METHODS}

\section{Subjects of Study}

Of those hospitalized patients at Dankook University Hospital, this study examined the 280 adult smokers who had visited the smoking cessation clinic of the family medicine department in order to quit smoking from January 2013 to July 2014. This study defined the entire process from actual smoking cessation consultation and treatment to re-visiting an outpatient clinic as treatment compliance. Of those referred patients, the 145 patients were not registered due to the following reasons: (1) some of these patients had already been discharged

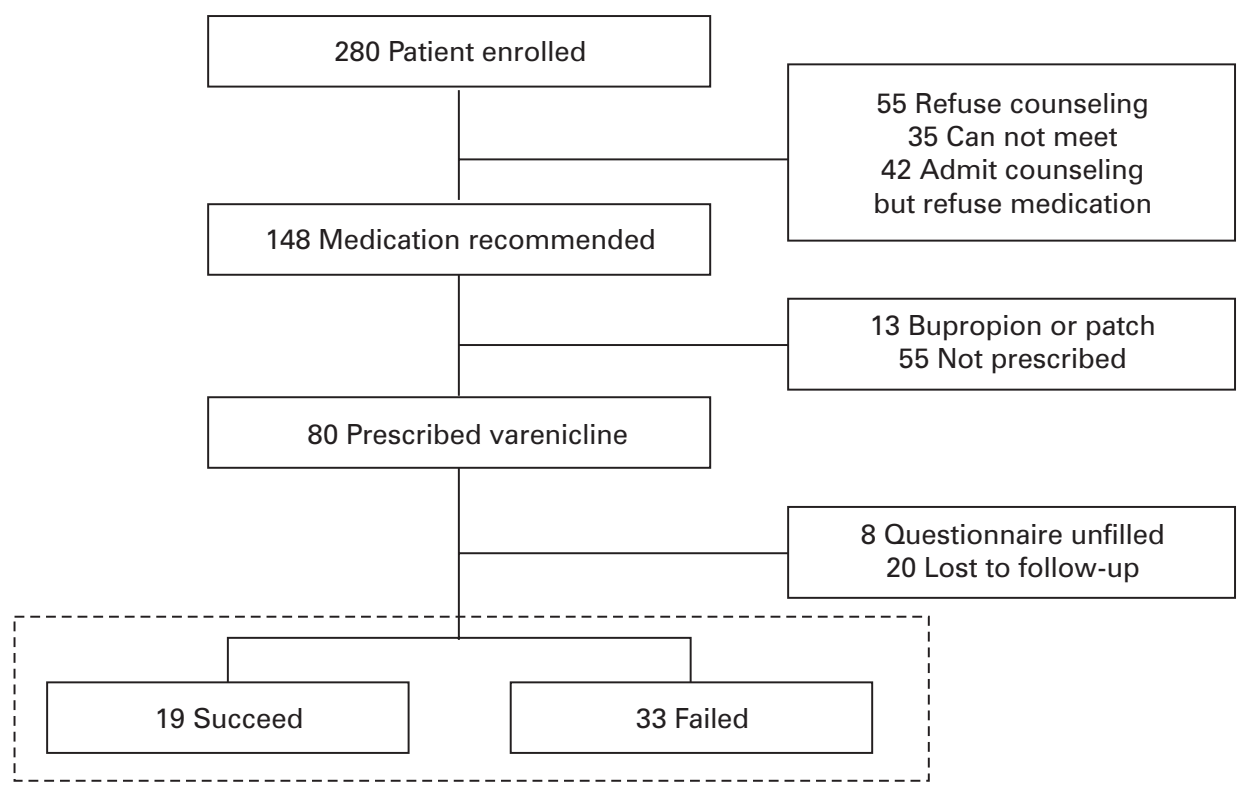

Figure 1. Flow chart for participants in the study. 
from the hospital before the smoking cessation consultation began, (2) some of these patients had severe disease so that they could not proceed with the consultation, and (3) some of these patients had refused to participate in the smoking cessation consultation. During the period of this study, a treatment request response that recommended to prescribe varenicline was created for a total of the 135 patients after the smoking cessation consultation. However, only the 80 patients actually received the prescription by their respective doctor. That is, only the 80 patients began the medication. In regard to these 80 patients, this study conducted the retrospective phone survey at least 6 months after the smoking cessation consultation ended for the last registered patient from January to February 2015. During this period, the 15 patients could not be reached over the phone even though we had tried to contact them at 5 times. Moreover, the 8 patients had not completed the first visit time questionnaire properly. The 3 patients had died during this period. The 2 patients had refused to participate in the phone survey. Therefore, this study has examined the smoking cessation rate and drug compliance of the 52 patients (Figure 1).

\section{Method of Study}

This study is a retrospective observational study. That is, this study was based on the phone survey conducted for one month from January 2015 and the self-report type survey data of the initial phase. Of the 80 prescribed patients, this study targeted the 52 patients. This study asked these 52 patients about whether they had successfully quit smoking for 6 months after being discharged from the hospital. This study also asked these 52 patients about the side-effects of drugs, the compliance, and the reasons why they had failed to quit smoking. This study defined those who had successfully quit smoking for 6 months after being discharged from the hospital as the 'successful group' who successfully quit smoking. For the other patients, this study defined them as the 'failed group.' In regard to the 35 patients who had spent more than 12 months after their first consultation, an annual smoking cessation rate was calculated. Furthermore, this study aimed to identify the association with the preliminary variables based on the survey data such as the demographic characteristics and smoking history between the successful group and the failed group. In regard to the demographic characteristics such as age, educational level, and monthly income level, which would influence the drug compliance, this study referred to the contents of the aforementioned self-report type questionnaires created at the time of consultation. For those patients who could not complete the questionnaire due to their medical condition at the time of hospitalization, they were instructed to fill up the short version of questionnaire that contained only the depression selective questions and smoking cessation willingness score. A total of the 12 patients had filled up this short version of questionnaire.

The main variables used in this analysis are as follows. First, in regard to Fagerstrom's Test for Nicotine Dependence that assesses the nicotine dependence in the self-report questionnaire of patients, a score of less than 3 points was classified as 'low dependence,' whereas a score of 4 to 6 points was classified as 'medium dependence,' and a score of more than 7 points was classified as 'high dependence.'

In regard to the willingness of patients for quitting smoking, this study conducted 10-point Likert scale in the beginning of the program with the question on whether they could completely succeed to quit smoking with this opportunity. A score of 1 to 3 points was classified as 'low degree of willingness,' whereas a score of 4 to 6 points was classified as 'intermediate degree of willingness,' and a score of 7 to 10 points was 'high degree of willingness.'

The drug compliance must have been based on a period of 12 weeks. However, the patients had usually received their prescription once a month. Furthermore, it was difficult to confirm whether they were actually taking medicine at the time of completing the 12-week program. Hence, this study inevitably had to define those patients who had taken the medicine as the drug compliance group based on the visit of 8th week visit. Also, this study defined the patients who had been prescribed varenicline from the family medicine department or their respective outpatient clinic more than once after they were discharged from the hospital.

\section{Overview of Hospitalized Smoking Cessation Program}

The hospitalized smoking cessation program of our hospital begins when relevant professors or attending physicians refer patients having agreed to quit smoking to the family medicine department among patients hospitalized at each clinical department. The list of referred patients is sent to the relevant professors of family medicine department. These patients will then be registered in the hospital computer system as a patient referred for smoking cessation. The patients who could move around freely were instructed to fill up a self-report questionnaire that contains the following questions after visiting the family medicine department: age, gender, educational level, monthly household income, marital status, weekly drinking quantity, weekly exercise frequency, nicotine dependence, willingness for quitting smoking, presence of depression, smoking period, smoking quantity (packyear), clinical department for hospitalization, name of disease at the time of hospitalization, and past experience for quitting smoking. These patients were then instructed to undergo the breath carbon monoxide measurement (Micro CO; Micro Medical Ltd., Chatham, $\mathrm{UK})$. The patients who were unable to move around freely were instructed to fill up a self-report questionnaire and undergo the carbon monoxide measurement in their hospital room after the relevant doctors of the family medicine department visited there. After conducting a one-on-one consultation with the patients based on the motivation elicitation theory, the doctors created a reply letter to recommend the patients to take the smoking cessation medicine during admission period, receive the additional prescription at the time of being discharged from the hospital, and make a reservation at the outpatient clinic of family medicine department.

Those patients who were suspected to suffer from depression or who were unable to proceed with oral administration were prescribed bupropion or nicotine patch. However, all of the other patients were prescribed varenicline. In the first week, they took $0.5 \mathrm{mg}$ of vareni- 
cline. For the first 3 days, they took it once a day. For the next 4 days, they took it twice a day. Since then, they took $1 \mathrm{mg}$ of varenicline twice a day. In regard to the prescription after discharge from the hospital, the patients were prescribed varenicline for 4 weeks unless there was an exceptional case. The average consultation time was about 20 to 40 minutes. No re-visit consultation was carried out unless there was an exceptional case.

\section{Statistical Analysis}

As for the statistical analysis, this study first summarized the data in an Excel file and then analyzed the data by utilizing PASW SPSS ver. 18.0 (SPSS Inc., Chicago, IL, USA). In regard to the univariate analysis, this study performed chi-square and t-test with significance level of 0.05. This study set the variables having a statistically significant difference in the univariate analysis as an independent variable. Moreover, this study performed logistic regression analysis with success of quitting smoking in 6 months as a dependent variable.

\section{RESULTS}

\section{General Characteristics and Treatment Compliance of Patients Referred for Smoking Cessation Treatment}

Of the 280 patients referred during the period, the 256 patients were men (91.4\%), whereas the 24 patients were women (8.6\%). The ages ranged from 19 years old to 86 years old. The average age was $56.3 \pm 12.8$ years old. The patients referred from the department of neurology accounted for the largest proportion with $60.4 \%$ (169 patients), followed by the department of cardiovascular medicine with 11.1\% (31 patients), the department of gastroenterology with 5.4\% (15 patients), and the department of orthopedics with $4.6 \%$ (13 patients).

Of the referred patients, the 190 patients received the consultation with the doctors of family medicine department. Sixty-seven point nine percent of the patients underwent the actual treatment based on their smoking cessation request. The 55 patients (19.6\%) refused to have an interview, whereas the 23 patients (8.2\%) were discharged from the hospital before the interview began. The 12 patients $(4.3 \%)$ were in a condition that they were unable to proceed with an interview. Of the 12 patients who were unable to proceed with an interview, the 11 patients (91.7\%) were hospitalized at the department of neurology. This study found no significant correlation between the refusal rate of interview, age, and hospitalization treatment of the patients.

Of the 190 patients who had an interview, the 135 patients (71.0\%) were recommended to take varenicline, whereas the 42 patients (22.1\%) had only an interview but were not prescribed any medicine. The 11 patients $(3.9 \%)$ were recommended to take bupropion, whereas the 2 patients $(0.7 \%)$ were recommended to take only nicotine patch. Of them, the 80 patients (59.3\%) were actually prescribed varenicline, whereas the 6 patients (54.5\%) were prescribed bupropion and the 1 patients (50.0\%) was prescribed nicotine patch.

Of the 80 patients who were prescribed varenicline at the time of hospitalization, the 43 patients (53.7\%) were additionally prescribed varenicline when they were discharged from the hospital. Of these 43 patients, only the 14 patients (32.5\%) were additionally prescribed varenicline by revisiting the outpatient clinic of family medicine department (Figure 1).

\section{General Characteristics and Smoking History of Varenicline Treatment Group}

Of the 52 subjects for the final analysis, the 50 patients were men (96.2\%), whereas only the 2 patients were women (3.8\%). The ages ranged from 19 years old to 78 years old. The average age was 52.1 years old. The 35 patients received the consultation in 2013, whereas the 17 patients received the consultation in 2014. The 30 patients were married people (75.0\%). The patients with a high school diploma accounted for the largest proportion with $42.5 \%$ (17 patients). The patients whose monthly household income was between 3 million Korean won (KRW) and 10 million KRW accounted for the largest proportion with $45.9 \%$ (17 patients out of 37 patients), followed by those whose monthly household income was between 1 million KRW and 3 million KRW with $43.2 \%$ (16 patients).

In regard to the clinical department of patients, the department of neurology accounted for the largest proportion with 36.5\% (19 patients), followed by the department of cardiology, the department of gastroenterology, the department of neurosurgery, and the department of orthopedics, all of which had the 5 patients (9.6\%) each. In regard to the disease, the stroke patients accounted for the largest proportion with $38.5 \%$ (20 patients), followed by the patients with cardio-

Table 1. Base line characters of final participants

\begin{tabular}{|c|c|}
\hline Characteristic & No. $(\%)$ \\
\hline \multicolumn{2}{|l|}{ Sex } \\
\hline Male & $50(96.2)$ \\
\hline Female & $2(3.8)$ \\
\hline \multicolumn{2}{|l|}{ Age (y) } \\
\hline$<40$ & $7(13.5)$ \\
\hline $41-50$ & $17(32.7)$ \\
\hline $51-60$ & $13(25.0)$ \\
\hline$\geq 61$ & $15(28.9)$ \\
\hline \multicolumn{2}{|l|}{ Education $(y, n=40)$} \\
\hline$<12$ & $30(75.0)$ \\
\hline$\geq 12$ & $10(25.0)$ \\
\hline \multicolumn{2}{|c|}{ Monthly household income (Korean won, $n=37$ ) } \\
\hline High $(\geq 6,000,000)$ & $3(8.2)$ \\
\hline Middle $(3,000,000-6,000,000)$ & $17(45.9)$ \\
\hline $\operatorname{Low}(<3,000,0000)$ & $17(45.9)$ \\
\hline \multicolumn{2}{|c|}{ Degree of dependence on nicotine $(n=44)^{*}$} \\
\hline High & $18(40.9)$ \\
\hline Medium & $18(40.9)$ \\
\hline Low & 8 (18.2) \\
\hline \multicolumn{2}{|l|}{ Willingness to quit $(n=49)^{\dagger}$} \\
\hline High & $19(38.8)$ \\
\hline Medium & $15(30.6)$ \\
\hline Low & $15(30.6)$ \\
\hline
\end{tabular}

*Nicotine dependency is acquired from Fagerstrom test for nicotine dependency. tWillingness is acquired from Likert scales. 
vascular disease with 9.6\% (5 patients: 2 patients with angina and 3 patients with myocardial ischemia) and the patients with a fracture with $5.8 \%$ (3 patients).

There were the 23 patients (44.2\%) who had voluntarily visited the smoking cessation clinic, whereas there were the 12 patients $(23.1 \%)$ who had visited the smoking cessation clinic at the recommendation of their attending physician. The 6 patients (11.5\%) answered that they fell in both of the aforementioned cases. Of the 42 respondents, the 26 patients $(61.9 \%)$ said that they had previously attempted to quit smoking. The average age of starting smoking was $20.5 \pm 4$ years old. It ranged from 14 years old to 32 years old. The average total smoking period was $31.4 \pm 12$ years. It ranged from 5 years to 60 years. The mean score of the nicotine dependence of the participants from the Fagerstrom questionnaire was $5.5 \pm 2$ points (Table 1 ).

\section{Drug Compliance, Reason for Cessation and Side-Effects in Relation to Varenicline}

Of the 52 subjects who were prescribed varenicline, the 2 patients (3.8\%) had never taken the medicine for a single day. Of the 52 patients who had taken the medicine for more than one day, the 9 patients (17.3\%) had taken the medicine for more than 8 weeks, whereas the 7 patients (13.5\%) had taken the medicine for more than 12 weeks. In regard to the reasons why they had stopped taking varenicline, the 13 patients $(28.9 \%)$ stopped taking it because they thought that they could quit smoking on their own will, whereas the 10 patients $(22.2 \%)$ stopped taking it because they had some side-effects. Also, the 8 patients (17.8\%) stopped taking it because they were not prescribed additional varenicline during their respective treatment process. Of the 50 patients who had taken varenicline, a total of the 12 patients $(24.0 \%)$ complained about the side-effects. Specifically, the 6 patients $(12.0 \%)$ had nausea, whereas the 3 patients $(6.0 \%)$ had dyspepsia. The 8 patients $(66.7 \%)$ stopped suffering from the side-effects when they stopped taking the medicine.

\section{Success Rate of Smoking Cessation and Related Factors Thereof}

Of the 52 patients, the 19 patients (36.5\%) had stopped smoking for more than 6 months. This study confirmed that the 15 patients $(42.8 \%)$ had stopped smoking for more than one year out of the 35 patients who had spent more than one year after the consultation.

In regard to each clinical department, the 11 patients (57.9\%) out of the 19 patients hospitalized at the department of neurology have succeeded to quit smoking for 6 months. Among the 33 patients from all of the other departments, the 8 patients $(24.2 \%)$ have succeeded to quit smoking. Hence, the success rate of smoking cessation of the patients hospitalized at the department of neurology was significantly higher $(\mathrm{P}=0.02)$. For each disease, the 11 patients (55.0\%) out of the 20 stroke patients have succeeded to quit smoking, whereas the 8 patients (25.0\%) out of the 32 patients with other diseases have succeeded to quit smoking. Hence, the success rate of smoking cessation of the stroke patients had a significantly higher success rate of smoking ces-
Table 2. Patient-related factors for success of 6 month cessation

\begin{tabular}{lrrrr}
\hline & Total & Success group & Failure group P-value* \\
\hline Admission department & & & 0.02 \\
$\quad$ Neurology & $19(100.0)$ & $11(57.9)$ & $8(42.1)$ & \\
$\quad$ Others & $33(100.0)$ & $8(24.2)$ & $25(75.8)$ & \\
Disease & & & 0.03 \\
$\quad$ Brain stroke & $20(100.0)$ & $11(55.0)$ & $9(45.0)$ & \\
$\quad$ Others & $32(100.0)$ & $8(25.0)$ & $24(75.0)$ & \\
Monthly income (Korean won) & & & & 0.05 \\
$\quad$ High & $3(100.0)$ & $1(33.3)$ & $2(66.7)$ & \\
$\quad$ Middle & $17(100.0)$ & $10(58.8)$ & $7(41.2)$ & \\
$\quad$ Low & $17(100.0)$ & $3(17.6)$ & $14(82.4)$ & \\
Medication (wk) & & & & 0.29 \\
$\geq 8$ & $11(100.0)$ & $2(18.2)$ & $9(81.8)$ & \\
$<8$ & $41(100.0)$ & $17(41.5)$ & $24(58.5)$ & \\
\hline
\end{tabular}

Values are presented as number (\%).

*From chi-square test for outcomes comparing a difference among 2-3 groups.

sation $(\mathrm{P}=0.03)$.

There was no significant difference in the success rate of smoking cessation depending on the age, reason for visiting the smoking cessation clinic, nicotine dependence stated in the preliminary questionnaire, daily smoking quantity, willingness of smoking cessation, smoking period, and revisiting family medicine department of the patients. Of the 10 patients who had taken the medicine for a long time (more than 8 weeks), the 2 patients (20.0\%) have succeeded to quit smoking for 6 months. Of the 42 patients who had taken within 8 weeks, the 17 patients $(40.5 \%)$ have succeeded to quit smoking. However, there was no significant correlation $(\mathrm{P}>0.29)$.

Marital status was not significantly correlated to smoking cessation. However, the 11 patients (55.0\%) of the 20 smokers whose monthly household income was more than 3 million KRW have succeeded to quit smoking, whereas only the 8 patients $(25.0 \%)$ of the 32 smokers whose monthly household income was less than 3 million KRW have succeeded to quit smoking. Thus, the patients whose monthly household income was more than 3 million KRW had a significantly higher success rate of smoking cessation $(\mathrm{P}<0.03)$.

In regard to the physical change due to smoking cessation, no particular physical change accounted for the largest proportion with $57.9 \%$ (11 patients), followed by skin improvement with $26.3 \%$ (5 patients) and weight increase with $21.1 \%$ (4 patients) (Table 2).

This study conducted the logistic regression analysis with age, referring department, disease causing hospitalization, and personal wealth as an independent variable and success of smoking cessation for 6 months as a dependent variable. However, there was no significant correlation.

\section{DISCUSSION}

This study indicated that there were various obstacles in the treatment process in terms of referring hospitalized patients to the smoking cessation clinic. Only about $67.9 \%$ of the aforementioned referred patients 
would actually have a consultation and prescription. Moreover, even those patients who were prescribed a medicine to quit smoking through a consultation have rarely revisited the outpatient clinic after discharge from the hospital due to the following reasons: a lack of willingness to quit smoking, discomfort of treatment procedure, and ignorance of relevant attending physicians. In particular, the proportion of those who had refused to have an interview was $19.6 \%$. This finding indicates that the patients were referred even when they were not fully aware of the need for the treatment to quit smoking.

Many domestic and overseas studies have proved that smoking cessation education and consultation for hospitalized patients were effective in increasing the success rate of smoking cessation. However, the previous studies on the smoking cessation of hospitalized patients have mainly focused on the effects of education and consultation., ${ }^{9,10)}$ Lee et al. ${ }^{11)}$ reported that the success rate of smoking cessation had improved significantly due to a simple recommendation for quitting smoking to hospitalized smokers. This study examined the drug compliance and relevant factors of varenicline that had been increasingly prescribed in the clinics. The proportion of the patients who were taking varenicline for more than 8 weeks was $17.3 \%$. Of these patients, the proportion of the patients who were taking for more than 12 weeks was only $13.5 \%$. This finding was lower than the result of the US study that the compliance after hospitalization treatment for one month was $56.0 \% .{ }^{12)}$ However, this finding was higher than the result of the study of Song et al. ${ }^{13)}$ that was $7.7 \%$ for 12 weeks.

The effect of varenicline on smoking cessation has already been proved on numerous occasions. The studies having covered the success rate of smoking cessation with the help of varenicline among Asian smokers have reported that the success rate for 6 months was $37.7 \%$ to $50.3 \%{ }^{6-8)}$ The study published by the South Korean primary medical treatment center in 2012 also reported that the success rate of smoking cessation for 7 days in the 6th month among the patients who had taken the medicine for more than 12 weeks was $63.6 \%{ }^{14)}$

However, this study found that there was no significant difference between the drug period of varenicline and the success rate of smoking cessation. In regard to the usability of varenicline for hospitalized patients, the previous studies had not found a significant result either. ${ }^{9)}$ The US study that had prescribed varenicline to the hospitalized patients in 2011 reported that there was no significant difference in the success rate of smoking cessation between the group having taken varenicline and the placebo group. ${ }^{12)}$ The meta analysis of 2008 showed an increase of $47.0 \%$ in the nicotine alternative treatment accompanied by the consultation treatment for the hospitalized patients. ${ }^{9)}$ However, it was not statistically significant. As such, there has been almost no study that attempted to compare only the effects of drug therapy in relation to the smoking cessation of hospitalized patients. Similarly, this study can hardly prove the validity of its results because the degree of drug compliance is very low in this study.

The success rate of smoking cessation for 6 months in this study is relatively high at $36.5 \%$. The reason was probably because the proportion of patients with severe disease such as stroke was high. The study conducted by Suner-Soler et al. ${ }^{15)}$ reported that the success rate of smoking cessation one year after stroke treatment was $40.0 \%$. The fact that the success rate of smoking cessation was high despite a low degree of drug compliance indicates that the effects of education and consultation are so huge as is commonly supposed. In this regard, it is imperative to introduce a systematic smoking cessation program that emphasizes the relationship between smoking and disease for hospitalized patients. This program offered the hospitalized patients a consultation only once during their hospitalization period unless there was an exceptional case. It was because there was a serious shortage of staffs. On that account, it is imperative for the government to devise a measure to support smoking cessation specialized nurses or doctors.

The proportion of the patients having experienced the side-effects of varenicline was $24.0 \%$. This was similar to the findings of Song et al. $^{13)}(32.3 \%)$ and Lee et al. ${ }^{14)}(30.8 \%)$. That nausea was the most frequently experienced side-effect was also consistent with the findings of the other studies. However, it was very low compared to the findings of Wang et al. ${ }^{6)}(77.0 \%)$, Nakamura et al. ${ }^{7)}(79.1 \%)$, and Tsai et al. ${ }^{8)}$ (86.5\%), which have targeted the Asian smokers. There is a possibility that weak symptoms were not verified due to the differences in the treatment and research environment.

This study also showed that such factors as hospitalization department, disease causing hospitalization, and monthly household income of patients had a high correlation with the success rate of smoking cessation. As for the hospitalization department, the department of neurology accounted for the largest proportion with $36.5 \%$. Most of the patients were stroke patients. Thus, the success rate of smoking cessation among the patients referred from the department of neurology was significantly higher than the patients referred from the other departments because the severity of disease might have a significant impact. Meanwhile, it could also be interpreted that the high referring rate itself reflected the high degree of interest of a certain clinical department toward smoking cessation.

That the success rate of smoking cessation is proportional to monthly household income has been often found in the previous studies. Such factors as age, educational level, marital status, and nicotine dependence were not significantly correlated to the success rate of smoking cessation. The previous study showed that the success rate of smoking rate became higher with age increase. ${ }^{13)}$ Some other studies have also pointed out the correlation between age and success rate of smoking cessation. ${ }^{14)}$ However, it was not significant in the other study that examined the hospitalized patients in 2009. ${ }^{10)}$ In this study, there was no correlation between marital status and success rate of smoking cessation. This finding is consistent with the results of the other domestic study. ${ }^{10)}$ In regard to smoking quantity and nicotine dependence, the findings of this study are similar to the success rate of smoking cessation found in the study of Song et al. ${ }^{13)}$ However, it was not significant in the other study that examined the hospitalized patients. ${ }^{10)}$ Thus, it would be imperative to consider the different characteristics of each patient.

In the study of Jung et al. ${ }^{10)}$ that was conducted with the hospitalized 
patients, those patients who decided to quit smoking at the recommendation of their respective doctor had a substantially higher success rate of smoking cessation than those who decided to quit smoking based on their own will. In the other studies, patient education of a doctor was deemed as an important influencing factor of smoking cessation. ${ }^{9)}$ In this study, the 4 doctors have proceeded with their respective consultation. Thus, there is a possibility that each doctor had different consultation contents.

The limitation of this study is a small number of samples. Therefore, it is hard to secure the verifiability of significant level. Furthermore, this study had only two women. Thus, this study could not discuss on the significant differences depending on gender. Also, this study was proceeded as a retrospective study. Thus, it may have an issue associated with recall bias. Add to this, some patients received the call more than one year after they were prescribed the medicine. This study may have an issue associated with the memory accuracy of patients because many patients of this study had reached a ripe old age or suffered from stroke.

In addition, the patients in this study were not instructed to bring the remaining pills or mark the number of days for which they had taken the medicine in an effort to assess their drug compliance. Instead of that, the patients were then instructed to tell the information over the phone. This assessment practice was certainly a limitation of this study. That is, some patients expressed that they had taken all the prescribed medicines. Therefore, there was a difficulty with measuring the date of taking the medicine objectively. The success of smoking cessation was also confirmed only over the phone. Thus, there is a possibility that the reliability of the data in this study for the actual success of smoking cessation is lower than such objective data as re-measurement of breath carbon monoxide.

The previous studies have mainly examined the success of smoking cessation and the drug compliance for the smokers having visited an outpatient clinic. In contrast, this study examined the treatment and drug compliance for the hospitalized patients for the first time in South Korea. Thus, this study is of great academic significance. Even though the family medicine department proceeds with a smoking cessation consultation for patients and sent a reply opinion of treatment request for prescription, the relevant clinical departments often ignored this prescription request. Moreover, many patients did not even make a reservation again at the outpatient clinic of family medicine department after discharge from the hospital. Therefore, this study showed the importance of cooperation with the medical staffs of each clinical department in order to enhance the treatment compliance of hospitalized smoking cessation program. Active smoking cessation treatment for hospitalized patients should be taken into consideration as a top priority in the smoking cessation policy.

\section{CONFLICT OF INTEREST}

No potential conflict of interest relevant to this article was reported.

\section{REFERENCES}

1. U.S. Department of Health and Human Services. How tobacco smoke causes disease: the biology and behavioral basis for smoking-attributable disease: a report of the surgeon general. Atlanta (GA): U.S. Department of Health and Human Services, Centers for Disease Control and Prevention, National Center for Chronic Disease Prevention and Health Promotion, Office on Smoking and Health; 2010.

2. Korean Ministry of Health and Welfare. The fifth Korea National Health and Nutrition Examination Survey, 2013. Sejong: Korean Ministry of Health and Welfare; 2013.

3. Pederson LL, Wanklin JM, Lefcoe NM. The effects of counseling on smoking cessation among patients hospitalized with chronic obstructive pulmonary disease: a randomized clinical trial. Int J Addict 1991; 26:107-19.

4. Hennrikus DJ, Lando HA, McCarty MC, Klevan D, Holtan N, Huebsch JA, et al. The TEAM project: the effectiveness of smoking cessation intervention with hospital patients. Prev Med 2005;40:249-58.

5. Stewart RB, Caranasos GJ. Medication compliance in the elderly. Med Clin North Am 1989;73:1551-63.

6. Wang C, Xiao D, Chan KP, Pothirat C, Garza D, Davies S. Varenicline for smoking cessation: a placebo-controlled, randomized study. Respirology 2009;14:384-92.

7. Nakamura M, Oshima A, Fujimoto Y, Maruyama N, Ishibashi T, Reeves KR. Efficacy and tolerability of varenicline, an alpha4beta2 nicotinic acetylcholine receptor partial agonist, in a 12-week, randomized, placebo-controlled, dose-response study with 40-week follow-up for smoking cessation in Japanese smokers. Clin Ther 2007;29:1040-56.

8. Tsai ST, Cho HJ, Cheng HS, Kim CH, Hsueh KC, Billing CB Jr, et al. A randomized, placebo-controlled trial of varenicline, a selective alpha4beta2 nicotinic acetylcholine receptor partial agonist, as a new therapy for smoking cessation in Asian smokers. Clin Ther 2007;29:1027-39.

9. Rigotti NA, Munafo MR, Stead LF. Smoking cessation interventions for hospitalized smokers: a systematic review. Arch Intern Med 2008;168: 1950-60.

10. Jung KY, Yoo SH, Ma SH, Hong SH, Lee YS, Shim UY, et al. Inpatient smoking cessation program and its success rate for abstinence among Korean smokers. Korean J Fam Med 2009;30:503-10.

11. Lee H, Lee BK, Bak SJ, Cho EJ, Kim MY, Yoon JL. The difference of smoking rates before and after hospital admission. Korean J Fam Med 2006;27:556-60.

12. Steinberg MB, Randall J, Greenhaus S, Schmelzer AC, Richardson DL, Carson JL. Tobacco dependence treatment for hospitalized smokers: a randomized, controlled, pilot trial using varenicline. Addict Behav 2011;36:1127-32.

13. Song B, Yun WS, Choi EY, Cheong YS, Park EW. Smoking cessation rate and related factors of varenicline in clinical practice. Korean J Fam Med 2011;32:112-9.

14. Lee JY, Kim MJ, Jun HJ, Kang M, Park AR, Oh DE, et al. Adherence to varenicline and abstinence rates for quitting smoking in a private health promotion center-based smoking cessation clinic. Tuberc Respir Dis 2012;72:426-32.

15. Suner-Soler R, Grau A, Gras ME, Font-Mayolas S, Silva Y, Davalos A, et al. Smoking cessation 1 year poststroke and damage to the insular cortex. Stroke 2012;43:131-6. 